OPEN ACCESS

Edited by:

Yoshiaki Suzuki,

Nagoya City University, Japan

Reviewed by:

Antonios Pantazis,

Linköping University, Sweden

Yu Zhou,

Washington University School of Medicine in St. Louis, United States

Kazuharu Furutani,

University of California, Davis,

United States

Rikard Blunck,

Université de Montréal, Canada

${ }^{*}$ Correspondence:

Jianmin Cui

jcui@wustl.edu

Specialty section:

This article was submitted to Membrane Physiology and Membrane Biophysics,

a section of the journal

Frontiers in Physiology

Received: 21 August 2021 Accepted: 01 October 2021

Published: 21 October 2021

Citation:

Cui J (2021) BK Channel Gating Mechanisms: Progresses Toward a Better Understanding of Variants

Linked Neurological Diseases.

Front. Physiol. 12:762175.

doi: 10.3389/fphys.2021.762175

\section{BK Channel Gating Mechanisms: Progresses Toward a Better Understanding of Variants Linked Neurological Diseases}

\author{
Jianmin Cui*
}

Department of Biomedical Engineering, Center for the Investigation of Membrane Excitability Disorders, Cardiac Bioelectricity and Arrhythmia Center, Washington University, St. Louis, MO, United States

The large conductance $\mathrm{Ca}^{2+}$-activated potassium $(\mathrm{BK})$ channel is activated by both membrane potential depolarization and intracellular $\mathrm{Ca}^{2+}$ with distinct mechanisms. Neural physiology is sensitive to the function of BK channels, which is shown by the discoveries of neurological disorders that are associated with BK channel mutations. This article reviews the molecular mechanisms of BK channel activation in response to voltage and $\mathrm{Ca}^{2+}$ binding, including the recent progress since the publication of the atomistic structure of the whole BK channel protein, and the neurological disorders associated with BK channel mutations. These results demonstrate the unique mechanisms of BK channel activation and that these mechanisms are important factors in linking BK channel mutations to neurological disorders.

Keywords: BK channel, voltage, calcium, activation, neurological disorders, loss of function mutation, gain of function mutation

\section{INTRODUCTION}

BK channels are activated by membrane depolarization and intracellular $\mathrm{Ca}^{2+}$ binding. Due to its large single channel conductance of 100-300 pS (Latorre et al., 1989), which gives rise to its name as the big $\mathrm{K}^{+}(\mathrm{BK})$ channel, the opening of the channel effectively repolarizes the membrane and stops $\mathrm{Ca}^{2+}$ from entering the cell due to deactivation of voltage gated $\mathrm{Ca}^{2+}$ channels (Lancaster and Nicoll, 1987; Storm, 1987). Therefore, BK channels are important in controlling cellular excitation and $\mathrm{Ca}^{2+}$ homeostasis. In 2005, a mutation of the BK channel was found to associate with epilepsy and movement disorder in human patients (Du et al., 2005). The mutation in the KCNMA1 gene that encodes the Slo1 $\alpha$-subunit of BK channels causes a missense change, D434G. This mutation alters $\mathrm{Ca}^{2+}$ dependent activation of the channel, resulting in an enhanced $\mathrm{Ca}^{2+}$ sensitivity ( $\mathrm{Du}$ et al., 2005; Díez-Sampedro et al., 2006; Lee and Cui, 2009; Yang et al., 2010). With the progress in human genetics, more KCNMA1 variants that link to neurological disorders have been identified. Some of the mutations in BK channels due to these variants have been functionally characterized, and the results show that these mutations alter voltage and $\mathrm{Ca}^{2+}$ dependent activation to different effects (Bailey et al., 2019; Miller et al., 2021). These results demonstrate that the changes in voltage and $\mathrm{Ca}^{2+}$ dependent activation of BK channels are important factors linking the KCNMA1 variants to neurological disorders.

Voltage and $\mathrm{Ca}^{2+}$ dependent activation of BK channels has been studied intensively since the discovery of BK channels in early 1980's (Marty, 1981; Pallotta et al., 1981). During the course 
of these studies, the DNA sequence of Slo1 was identified (Atkinson et al., 1991; Adelman et al., 1992; Butler et al., 1993) and the atomistic structures of the channel protein were solved. The first atomistic structure data came from MthK, a $\mathrm{K}^{+}$channel that lacks the voltage sensor but has a cytosolic structure resembling that of BK channels (Jiang et al., 2002a,b). The cytosolic structure of BK channels was subsequently solved using X-ray crystallography (Wu et al., 2010; Yuan et al., 2010; Yuan et al., 2012), and recently the structure of the whole BK channel with and without the association of the modulatory $\beta 4$ subunit was solved using cryo-EM (Hite et al., 2017; Tao et al., 2017; Tao and MacKinnon, 2019). Each of these structural discoveries has revealed a new dimension in the organization of the molecular components important for voltage and $\mathrm{Ca}^{2+}$ dependent activation, allowed the use of additional approaches to explore the mechanisms, and as a result, led to a leap of understanding. In this article I will first review the studies prior to the publication of cryo-EM structures of BK channels, which have defined the important frameworks for understanding BK channel activation. The cryo-EM structures of the whole BK channel have revealed the interactions among structural domains with and without $\mathrm{Ca}^{2+}$ binding (Hite et al., 2017; Tao et al., 2017; Tao and MacKinnon, 2019) that are important for understanding the couplings of $\mathrm{Ca}^{2+}$ and voltage sensors to the opening of the pore. These structures also help reveal novel mechanisms of BK channel activation that differ from canonical mechanisms that were known in other $\mathrm{K}^{+}$channels. Finally, the changes in voltage and $\mathrm{Ca}^{2+}$ dependent activation with some variants linked to neurological diseases will be described, and the relationship between the change of molecular mechanisms and clinical presentations will be discussed.

\section{Established Frameworks for Understanding BK Channel Activation}

Ion channel activation involves three major molecular processes: sensor activation, sensor-pore coupling, and pore opening. Sensors in channel proteins change conformation upon the stimulation of signals, such as changes in the membrane potential and ligand binding. The conformational change of sensors is propagated to the pore via interactions between sensors and the pore, known as sensor-pore coupling. Finally, the pore opens to allow ionic flow across the membrane. BK channel activation depends on both membrane potential and intracellular $\mathrm{Ca}^{2+}$. The studies of BK channel activation have revealed the following frameworks for understanding how the two stimuli open the same pore.

First, $\mathrm{Ca}^{2+}$ and voltage activate the channel with distinct mechanisms. In early studies the relation between $\mathrm{Ca}^{2+}$ and voltage in activating BK channels was an important question. The identification of Slo1 gene KCNMA1 and the availability of cDNA of Slo1 for functional expression of BK channels in exogenous cells (Atkinson et al., 1991; Adelman et al., 1992; Butler et al., 1993) allowed the studies to distinguish the distinct $\mathrm{Ca}^{2+}$ and voltage dependent activation mechanisms (Cui et al., 1997). When the intracellular $\mathrm{Ca}^{2+}$ concentration was kept low $(\leq 0.5 \mathrm{nM})$ BK channels opened in response to membrane depolarization with a rate that exceeded the diffusion limit for $\mathrm{Ca}^{2+}$ to bind, suggesting that the channel can open by voltage without $\mathrm{Ca}^{2+}$ binding (Cui et al., 1997; Figures 1A,B). On the other hand, when the voltage sensor was kept at the resting state by negative membrane potentials $(\leq-140 \mathrm{mV}$ ) (Horrigan et al., 1999; Cui and Aldrich, 2000) the open probability of BK channels increased by 4 orders of magnitude when $\mathrm{Ca}^{2+}$ concentration was elevated to $100 \mu \mathrm{M}$ (Horrigan and Aldrich, 2002; Yang et al., 2010; Figures 1C,D), suggesting that the channel can open by $\mathrm{Ca}^{2+}$ binding without voltage. Subsequent studies identified the voltage sensor and $\mathrm{Ca}^{2+}$ binding sites in different structural domains of the channel. While the residues responsible for voltage sensing are located in the transmembrane segments (Diaz et al., 1998; Cui and Aldrich, 2000; Ma and Horrigan, 2005; Pantazis et al., 2010a; Zhang et al., 2014), the $\mathrm{Ca}^{2+}$ binding sites are found to reside in the cytosolic domain (Schreiber and Salkoff, 1997; Shi et al., 2002; Xia et al., 2002; Bao et al., 2004; Yusifov et al., 2008; Wu et al., 2010; Yuan et al., 2010; Zhang et al., 2010; Javaherian et al., 2011; Yuan et al., 2012; Hite et al., 2017; Tao et al., 2017; Tao and MacKinnon, 2019). Thus, voltage and $\mathrm{Ca}^{2+}$ activate the channel by perturbing different structure domains that harbor the respective sensors and can open the channel independent of each other.

Second, $\mathrm{Ca}^{2+}$ and voltage activate the channel with allosteric mechanisms. In the extreme experimental conditions with low $\mathrm{Ca}^{2+}$ concentrations $(\leq 0.5 \mathrm{nM})$ and negative membrane potentials $(\leq-140 \mathrm{mV})$ BK channels were observed to open with a small but measurable open probability $\left(\sim 10^{-7}\right.$ ) (Horrigan et al., 1999; Cui and Aldrich, 2000; Figure 1C). In these experimental conditions the channel opening was not controlled by $\mathrm{Ca}^{2+}$ binding or voltage sensor activation, but was an intrinsic spontaneous event. The open probability of BK channels increases with $\mathrm{Ca}^{2+}$ binding or voltage sensor activation, since at the open conformation the channel has a higher $\mathrm{Ca}^{2+}$ affinity and a facilitated voltage sensor activation (McManus and Magleby, 1991; Cox et al., 1997; Horrigan et al., 1999; Horrigan and Aldrich, 2002). Each BK channel contains eight high-affinity $\mathrm{Ca}^{2+}$ binding sites (Schreiber and Salkoff, 1997; Shi et al., 2002; Xia et al., 2002; Bao et al., 2004; Wu et al., 2010; Yuan et al., 2010, 2012; Zhang et al., 2010; Hite et al., 2017; Tao et al., 2017; Tao and MacKinnon, 2019) and four voltage sensors (Hite et al., 2017; Tao et al., 2017; Tao and MacKinnon, 2019; Figure 2), and the open probability of the closed-open transitions increases with each $\mathrm{Ca}^{2+}$ binding and voltage sensor activation. This mechanism of $\mathrm{Ca}^{2+}$ and voltage dependent activation can be quantitatively described by a model that accounts for BK channel activation with the changes of $\mathrm{Ca}^{2+}$ concentration and voltage at a broad range that includes and extends beyond physiological conditions (Horrigan and Aldrich, 2002; Figure 2A). This mechanism is in contrast to Shaker $\mathrm{K}^{+}$channels, for which the opening was tightly controlled by voltage sensor activation even when the open probability was at $10^{-7}$ (Islas and Sigworth, 1999). Thus, the mechanism of coupling between sensor activation and pore opening in Shaker $\mathrm{K}^{+}$channels is thought to be obligatory, while in BK channels is allosteric. The allosteric mechanism makes it possible for $\mathrm{BK}$ channels to sense both $\mathrm{Ca}^{2+}$ binding and voltage sensor activation for opening. In a channel with the pore tightly 

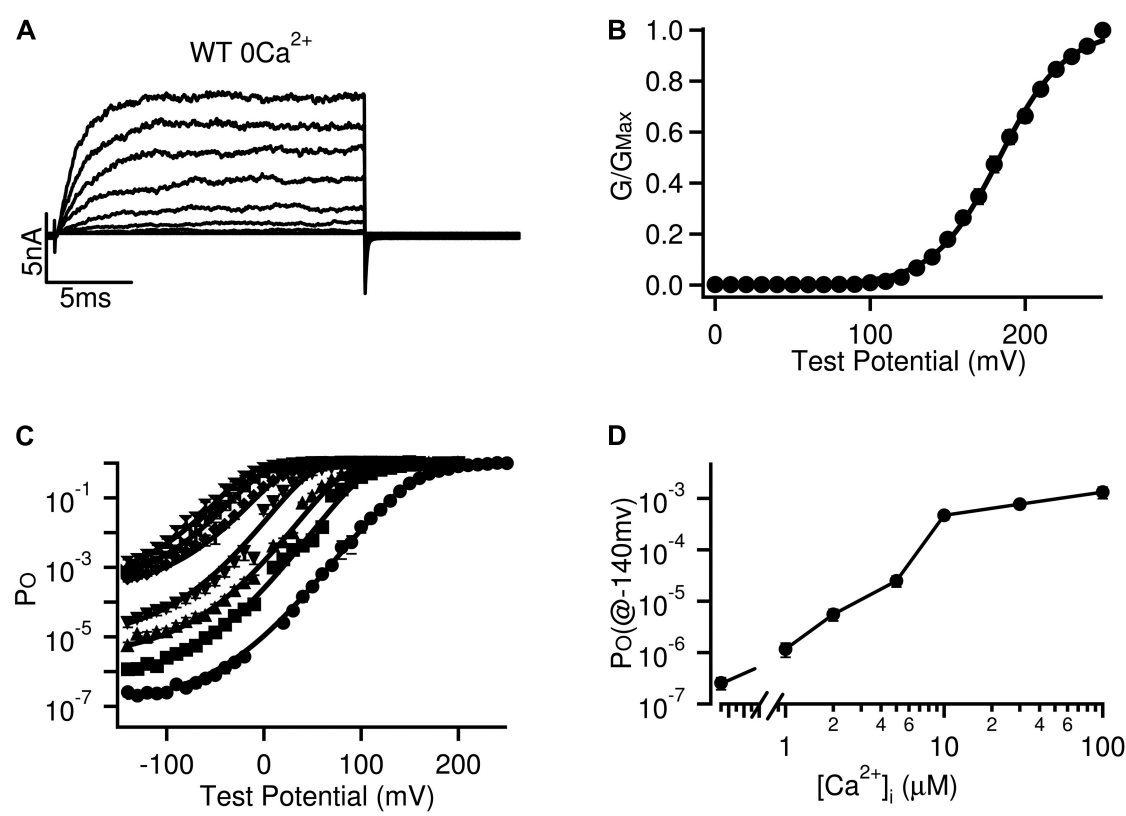

FIGURE 1 | Independent voltage and $\mathrm{Ca}^{2+}$ activation of BK channels. (A) Current traces activated by voltage pulses in low $\mathrm{Ca}^{2+}$ concentration ( $\leq 0.5 \mathrm{nM}$ ) without binding to $\mathrm{Ca}^{2+}$. (B) Steady state conductance of the channels (measured at the end of the current traces in panel (A)) at different voltages. The GV relation reflects voltage dependence of open probability of the channels. (C) Open probability of $\mathrm{BK}$ channels at different voltage and $\mathrm{Ca}^{2+}$ concentrations. At negative voltages $(\mathrm{V}<-100 \mathrm{mV})$ channels open in response to $\mathrm{Ca}^{2+}$ concentration changes without dependence on voltage sensor activation. (D) Dependence of open probability at $-140 \mathrm{mV}$ on $\mathrm{Ca}^{2+}$ concentration. The same results have been published previously (Yang et al., 2010).

controlled by the voltage sensor, such as Shaker, $\mathrm{Ca}^{2+}$ binding would not have been able to open the channel without voltage sensor activation.

Third, interactions among structural domains are important for $\mathrm{Ca}^{2+}$ and voltage dependent activation of BK channels (Figure 2B). The primary sequence of Slo1 channels, in comparison with voltage gated $\mathrm{K}^{+}(\mathrm{Kv})$ channels, indicated that the channel included three structural domains, the transmembrane pore-gate domain (PGD), the transmembrane voltage sensor domain (VSD), and the cytosolic domain (CTD) (Cui, 2010; Lee and Cui, 2010). The structure of MthK (Jiang et al., 2002a,b) and subsequently the structures of the BK CTD (Wu et al., 2010; Yuan et al., 2010; Yuan et al., 2012) showed that the CTD contains two RCK (Regulator of $\mathrm{K}^{+}$Conductance) domains in each Slo1 subunit, and the eight RCK domains of the four subunits form a ring-like structure called the gating ring. Based on the sequence homology with $\mathrm{Kv}$ channels, the structures of the VSD, which contains the transmembrane segments S1S4, and PGD, which contains S5-S6, were modeled after the structure of Kv1.2 (Long et al., 2005a; Lee and Cui, 2009). Two $\mathrm{Ca}^{2+}$ binding sites, the $\mathrm{Ca}^{2+}$ bowl that is primarily formed by the RCK2 residues (Schreiber and Salkoff, 1997; Bao et al., 2004; Yusifov et al., 2008; Wu et al., 2010; Hite et al., 2017; Tao et al., 2017; Tao and MacKinnon, 2019) and the site that is located in RCK1 (Shi et al., 2002; Xia et al., 2002; Wu et al., 2010; Zhang et al., 2010; Yuan et al., 2012; Hite et al., 2017; Tao et al., 2017; Tao and MacKinnon, 2019), were found in the CTD of each Slo1. Residues in the VSD that are important for voltage sensing were identified (Ma and Horrigan, 2005; Zhang et al., 2014). Thus, the VSD and CTD harbor the voltage and $\mathrm{Ca}^{2+}$ sensors and propagate the stimuli to open the pore in PGD via interactions among these structure domains.

\section{Domain-Domain Interactions for Sensor-Pore Couplings in BK Channel Activation}

Prior to the publication of the cryo-EM structures of the whole BK channel (Hite et al., 2017; Tao et al., 2017; Tao and MacKinnon, 2019; Figures 2B,C) the coupling of $\mathrm{Ca}^{2+}$ binding in the CTD to the opening of the PGD had been proposed to involve two types of interactions among the structure domains. A study Niu et al. (2004) showed that voltage and $\mathrm{Ca}^{2+}$ dependent activation of BK channels was changed by altering the covalent link between the PGD and the CTD via a peptide of 15 residues, known as the C-linker. The changes in activation depended on the changes in C-linker length by addition or deletion of amino acids in a relationship as if the interaction between the PGD and CTD were like pulling a spring for channel activation. Thus, it was proposed that $\mathrm{Ca}^{2+}$ may activate the channel by causing a conformational change in the CTD that directly tugs the pore to open via the C-linker. On the other hand, the CTD was proposed to also interact with the VSD via non-covalent interactions, and such interactions may indirectly open the pore via VSD-PGD interactions (Lee and Cui, 2009). This proposal derived from the finding that the residues from both the VSD and CTD form a $\mathrm{Mg}^{2+}$ binding site that modulates voltage dependent activation 

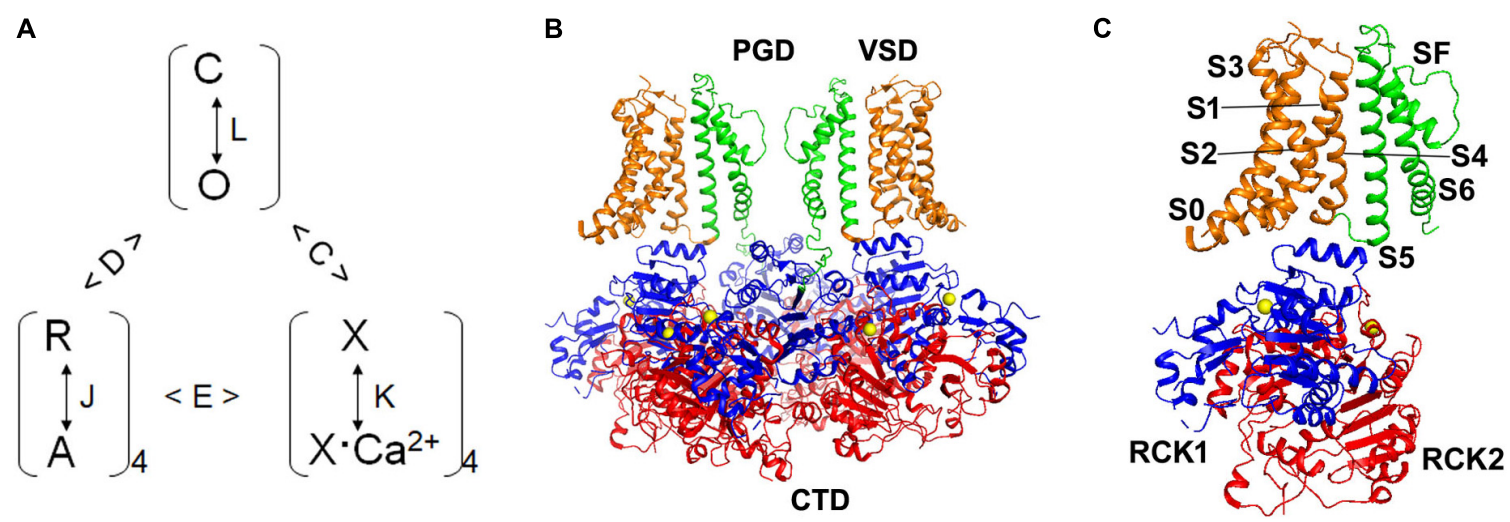

FIGURE 2 | Allosteric activation mechanism and structure of BK channels. (A) The allosteric model of BK channel activation. The channel undergoes an intrinsic closed-open transition ( $\mathrm{C}$ and $\mathrm{O})$, which is regulated by voltage sensor activation $(\mathrm{R}$ to $\mathrm{A})$ and $\mathrm{Ca}^{2+}$ binding $\left(X\right.$ to $\mathrm{X}$ :Ca $\left.{ }^{2+}\right)$ with allosteric mechanisms. i.e., with each voltage sensor activation the channel open state is favored by an allosteric factor $\mathrm{D}$; and with each $\mathrm{Ca}^{2+}$ binding to the channel the channel open state is favored by an allosteric factor C. L, J, and K are equilibrium constants for the respective transitions. Although voltage and $\mathrm{Ca}^{2+}$ can activate the channel independently, voltage sensor activation and $\mathrm{Ca}^{2+}$ binding affect each other with an allosteric factor $\mathrm{E}$. In this model only $4 \mathrm{Ca}^{2+}$ binding sites are assumed. Similar models containing 8 $\mathrm{Ca}^{2+}$ binding sites can also fit the electrophysiology data (Savalli et al., 2012). (B) BK channel structure with four Slo1 subunits (PDB entry: 6V38). Only two subunits are shown for the membrane spanning part of the channel. VSD: voltage sensing domain, PGD: pore-gate domain, CTD: cytosolic domain. (C) A single Slo1 subunit (PDB entry: 6V38). S0-S6: transmembrane segments, SF: selectivity filter, the two $\mathrm{Ca}^{2+}$ bound to the channel are shown in yellow.

(Shi and Cui, 2001; Hu et al., 2003; Zeng et al., 2005; Yang et al., 2007; Yang et al., 2008), and thus VSD and CTD are located closely. This mechanism was consistent with an earlier finding that the part of the CTD that is located close to the VSD is important in determining the different $\mathrm{Ca}^{2+}$ sensitivities between different BK channel homologs (Krishnamoorthy et al., 2005), and subsequent studies showed that alterations of the VSDCTD interactions indeed affected $\mathrm{Ca}^{2+}$ dependent activation (Yang et al., 2013; Geng et al., 2020).

For voltage dependent activation, it was found that a negatively charged residue E219 in the VSD, which contributed to voltage sensing, interacted with charged residues E321 and E324 in the PGD to mediate the coupling of voltage sensor activation to channel opening (Zhang et al., 2014). The interaction, however, was also found to be involved in the coupling between $\mathrm{Ca}^{2+}$ bindings and pore opening. Similar to $\mathrm{Ca}^{2+}$ dependent activation that involves interactions among all CTD, VSD, and PGD, voltage dependent activation also involves all three structure domains. A comparison between the voltage dependent activation of the wild type BK channel with that of a truncated BK channel with CTD deletion (Budelli et al., 2013) showed that the CTD was important in the coupling between VSD activation and pore opening (Zhang et al., 2017). The CTD undergoes conformational changes during voltage dependent activation as detected by fluorescence signals (Miranda et al., 2018). $\mathrm{Ca}^{2+}$ binding to the CTD also alters voltage dependence of channel activation (Horrigan and Aldrich, 2002; Sweet and Cox, 2008; Savalli et al., 2012; Lorenzo-Ceballos et al., 2019). These results suggest that the interactions among all three structure domains are involved in voltage and $\mathrm{Ca}^{2+}$ dependent activation.

The CTD-VSD non-covalent interactions were more clearly shown by the cryo-EM structures of the whole BK channels (Hite et al., 2017; Tao et al., 2017; Tao and MacKinnon, 2019; Figures $\mathbf{2 B}, \mathbf{C})$. The CTD gating ring is located closely to the membrane spanning domains of the channel, with an extensive interface between the N-terminus of the CTD with the cytosolic part of the VSD ( $585 \AA^{2}$ per subunit in the $\mathrm{Ca}^{2+}$ structure) (Hite et al., 2017). Comparing the structure with $\mathrm{Ca}^{2+} / \mathrm{Mg}^{2+}$ bound to the metal-free structure the N-lobes of the RCK1 domain, which face the VSD, tilted in a rigid body fashion away from the pore axis. In the $\mathrm{Ca}^{2+} / \mathrm{Mg}^{2+}$ bound structure the cytosolic part of the VSD also showed a corresponding outward displacement, and the movements of both the RCK1 and VSD alter the interface between these domains. This $\mathrm{Ca}^{2+}$ dependent change in the interactions between CTD and VSD suggests that these interactions are part of the coupling mechanism for $\mathrm{Ca}^{2+}$ binding to open the channel, and is consistent with the studies to show that mutations in the VSD-CTD interface alter $\mathrm{Ca}^{2+}$ sensitivity (Yang et al., 2013; Geng et al., 2020). The C-linker in the cryoEM structures is partly $\alpha$-helical and partly extended and nearly identical in both the structures with and without $\mathrm{Ca}^{2+} / \mathrm{Mg}^{2+}$ bound, but undergoes a large positional displacement laterally as a rigid unit. Recent studies showed that the C-linker can interact with the membrane directly to alter BK channel activation (Tian et al., 2019; Yazdani et al., 2020b). These results suggest that the C-linker may not affect channel activation simply as a passive link between the CTD and PGD, but directly interact with the membrane and other parts of the channel protein such as the gating ring to mediate $\mathrm{Ca}^{2+}$ and voltage dependent activation.

In the tetrameric structure of the Kv1.2 channel the VSD of each subunit is adjacent to the PGD of its neighboring subunit, showing a domain-swapped configuration (Long et al., 2005b). The BK channel, however, does not show such a domainswapping. Instead, the VSD and PGD of the Slo1 subunit interact within each subunit (Hite et al., 2017; Tao et al., 2017; Tao and MacKinnon, 2019; Figure 2C). This difference as revealed by the cryo-EM structure of BK channels suggests that the coupling between VSD activation to pore opening differs from 
the canonical electromechanical coupling mechanism for the domain-swapped Kv channels. In domain swapped $\mathrm{Kv}$ channels the peptide linking the VSD and the PGD of a subunit, the S4-S5 linker, interacts with the cytosolic part of the transmembrane helix S6 (Long et al., 2005b). S6 lines the inner pore, and the helices from four subunits cross at the cytosolic side of the membrane to form the activation gate that controls ionic flow during the opening and closing of these $\mathrm{Kv}$ channels (Liu et al., 1997; Long et al., 2005a,b). The interactions between the S4-S5 linker and S6 has been shown to mediate electromechanical coupling, which open the activation gate in response to VSD movements (Lu et al., 2001; Long et al., 2005b; Hou et al., 2020; Cowgill and Chanda, 2021). However, in BK channels, the S4-S5 linker of each subunit is short and not located close to S6, but interacts with the N-terminus of RCK1 (Hite et al., 2017; Tao et al., 2017; Tao and MacKinnon, 2019; Figure 2C). The VSD seems to be in close contact with the PGD within the same Slo1 subunit only with the interface between S4 and S5 helices (Figures 2B,C). The contact between S4 and S5 helices is extensive, which suggests that the interactions between the two helices are important for mediating the coupling between VSD activation and pore opening.

The extensive interactions between the S4 and S5 would also restrict the motion of $S 4$, which may be responsible for the unique VSD movements during BK channel activation. In domain swapped $\mathrm{Kv}$ channels the charged residues that sense voltage for channel activation, known as gating charges, are primarily found in the S4 transmembrane helix (Aggarwal and MacKinnon, 1996; Mannuzzu et al., 1996; Seoh et al., 1996). S4 moves across the membrane with a large distance upon membrane potential depolarization, resulting in a transfer of total 12-16 equivalent gating charges across the electric filed in the membrane (Zagotta et al., 1994a,b; Aggarwal and MacKinnon, 1996; Mannuzzu et al., 1996; Seoh et al., 1996; Bezanilla, 2000; Gandhi and Isacoff, 2002). However, in BK channels gating charges are found in S2 (D153 and R167), S3 (D186), and S4 (R213, E219) segments (Ma and Horrigan, 2005; Pantazis et al., 2010a; Zhang et al., 2014). During voltage dependent activation of BK channels a total 2.32 equivalent gating charges are moved across the electric filed in the membrane (Horrigan and Aldrich, 1999; Ma and Horrigan, 2005), much smaller as compared to those in domain swapped Kv channels. These results suggest that the VSD may undergo small movements that involve many transmembrane segments during voltage dependent activation (Ma and Horrigan, 2005; Pantazis et al., 2010a,b; Pantazis and Olcese, 2012; Pantazis et al., 2018). Furthermore, the pore opening in BK channels also retrospectively control VSD activation, such that VSD activation increases with multiple time courses, and some of which are in parallel with channel opening (Horrigan and Aldrich, 1999; Savalli et al., 2006), consistent with the idea that the S4-S5 interactions may restrict VSD movements during BK channel activation.

\section{Hydrophobic Gate in BK Channels}

Cryo-EM structures of BK channels reveal that the S6 helices from the four Slo1 subunits do not cross at the cytosolic side, either with or without $\mathrm{Ca}^{2+} / \mathrm{Mg}^{2+}$ bound (Figure $2 \mathrm{~B}$ ). If the metal-free structure represents the closed state of the channel, while the $\mathrm{Ca}^{2+} / \mathrm{Mg}^{2+}$ bound structure represents an open state (Hite et al., 2017), the cytosolic side of the pore is thus wide open at both the open and closed states of the channel. This result is consistent with the suggestion from the studies of BK channel block by quaternary ammonium (QA) molecules (Li and Aldrich, 2004; Wilkens and Aldrich, 2006; Tang et al., 2009) and other smaller molecules (Zhou et al., 2011; Zhou et al., 2015) that the channels do not have an intracellular gate so that the blockers can enter the pore even at the closed state. The study of a peptide blocker suggests that the cytosolic part of the pore may undergo a conformational change to reduce the pore size during BK channel closing ( $\mathrm{Li}$ and Aldrich, 2006), but the change is not sufficient to close the pore to restrict the entrance of $\mathrm{K}^{+}$ions or QA blockers. These results indicate that the activation gate of $\mathrm{BK}$ channels may be located above the cytosolic side, possibly at the selectivity filter, which is the narrowest part of the pore (Figures $\mathbf{2 B}, \mathbf{C}$ ) and with a conserved function among $\mathrm{K}^{+}$channels to select $\mathrm{K}^{+}$ion over other ions for permeation (Hite et al., 2017; Tao et al., 2017; Tao and MacKinnon, 2019).

Recently, an alternative mechanism for the activation gate was proposed. The structures of the metal free and $\mathrm{Ca}^{2+} / \mathrm{Mg}^{2+}$ bound BK channels suggested that the inner pore of the channel underwent a conformational change in the absence of metal binding, which mainly involved an amphipathic segment of S6, $\mathrm{V}_{319} \mathrm{PEIIE}_{324}$, to expose hydrophobic residues V319 and I323 to the pore inner surface (Hite et al., 2017; Tao et al., 2017; Tao and MacKinnon, 2019). As a result of these changes the inner pore beneath the selectivity filter becomes narrower, more elongated and hydrophobic. A molecular dynamics simulation study found that these changes promote dewetting transitions that completely deplete the inner pore of liquid water, giving rise to a vapor barrier to block the ion flow (Jia et al., 2018). Importantly, the dry pore remains physically open with an average diameter of $\sim 6 \AA$, allowing QA blockers to access the deep-pore region to block the channel even in the closed state. Such a hydrophobic gate depends on the hydrophobicity of the surface residues and the geometry of the inner pore (Aryal et al., 2015; Yazdani et al., 2020a). A functional study showed that the mutations in the BK channel inner pore A316D and A316V made the channel constitutively open and harder to open, respectively (Chen et al., 2014). Consistent with these results molecular dynamics simulations found that A316D reduced hydrophobicity of the pore and prevented dewetting transitions, while $\mathrm{A} 316 \mathrm{~V}$ enhanced hydrophobicity of the pore and made dewetting transitions faster with less water molecules remaining in the pore (Jia et al., 2018).

At present the hydrophobic gating mechanism is primarily based on molecular dynamic simulations, which can explain the experimental results of QA blockers on BK channel and some mutations. There has not been a direct experimental validation of this mechanism, partly due to the dilemma that once the vapor barrier forms the channel is closed and devoid of any functional detection. Comparing to the hydrophobic gate, the alternative mechanism, the selectivity filter acting as the activation gate, is not clearly defined with computational or experimental evidence. Where is the activation gate of BK channels and how it operates 
are not only part of the fundamental mechanism of voltage and $\mathrm{Ca}^{2+}$ dependent activation but also important for understanding BK channel modulation by compounds that act in the pore of BK channels, such as the QA blockers, paxilline (Zhou et al., 2020), and NS11021 (Rockman et al., 2020; Schewe et al., 2019). Some of these modulators have been excellent tools for the studies of the roles of BK channels in cellular and tissue function (Kaczorowski and Garcia, 2016; Cui, 2020), and may serve as the lead for therapies for BK variants linked neurological diseases as described in the following sections.

\section{Slo1 Mutation D434G Enhances $\mathrm{Ca}^{2+}$ Sensitivity and Is Associated With Epilepsy and Paroxysmal Non-kinesigenic Dyskinesia}

In a study of a family with inherited generalized epilepsy and coexistent paroxysmal dyskinesia (GEPD), a heterozygous A-G transition in exon 10 of KCNMA1 was identified, which results in the $\mathrm{D} 434 \mathrm{G}$ mutation in the $\mathrm{BK}$ channel subunit Slo1 (Du et al., 2005; Figure 3A). The inheritance followed an autosomal dominant pattern, and sixteen members of the family were affected. Among these individuals nine had early childhood onset of absence epilepsy, showing the characteristic episodes of loss of awareness, with vacant staring and unresponsiveness, and electroencephalography (EEG) showing synchronous spike-and-wave discharges (SWDs). Three of these individuals also developed generalized tonic-clonic seizures. The seizures in some of these individuals were responsive to anti-epilepsy medicine valproate, lamotrigine, or clonazepam. Among the sixteen affected individuals twelve had paroxysmal dyskinesia including five with both seizures and paroxysmal dyskinesia. These individuals were described to have involuntary dystonic or choreiform movements of the mouth, tongue, and extremities. The episodes were not induced by sudden movements, but induced by alcohol, fatigue, and stress, so that the paroxysmal dyskinesia was classified as paroxysmal non-kinesigenic dyskinesia (PNKD). It was noted that the episodes of PNKD developed around the same age as the onset of the seizures, but during the episodes the individual had preserved consciousness.

Comparison of the currents of the D434G BK channels with the wild type (WT) BK currents showed that the D434G currents were increased with a faster activation time course at the same voltage and intracellular $\mathrm{Ca}^{2+}$ concentrations $\geq 1 \mu \mathrm{M}$ (Du et al., 2005; Díez-Sampedro et al., 2006; Lee and Cui, 2009; Wang et al., 2009; Yang et al., 2010; Moldenhauer et al., 2020), which is the cytosolic $\mathrm{Ca}^{2+}$ concentration at neuronal excitations (Figures 3B,C). These results suggest that more currents flow through the D434G BK channels during an action potential. At these $\mathrm{Ca}^{2+}$ concentrations, the voltage dependence of channel conductance (GV relation) of D434G BK channels was shifted to more negative voltages (Figure $3 \mathrm{C}$ ), indicating that channel activation was increased. The increased channel activation was actually primarily because of an increased $\mathrm{Ca}^{2+}$ sensitivity without changes in voltage dependent activation, because the mutation increased the apparent affinity for $\mathrm{Ca}^{2+}$ in channel activation (Díez-Sampedro et al., 2006; Wang et al., 2009; Yang et al., 2010), while at low $\mathrm{Ca}^{2+}$ concentrations $(\sim 0.5 \mathrm{nM})$ with few $\mathrm{Ca}^{2+}$ binding to the channel the $\mathrm{D} 434 \mathrm{G}$ mutation did not alter GV relation (Díez-Sampedro et al., 2006; Yang et al., 2010). The residue D434 is located close to the $\mathrm{Ca}^{2+}$ binding site in RCK1 (Figure 3A). The mutation D434G neutralizes a negative charge close to the $\mathrm{Ca}^{2+}$ binding site and makes the protein structure more flexible around the site, which may be responsible for the mutation to specifically enhance the affinity of $\mathrm{Ca}^{2+}$ for the RCK1 site and strengthen the coupling between $\mathrm{Ca}^{2+}$ binding to the site with pore opening (Yang et al., 2010).

Recently a knock-in mouse model carrying BK D434G mutation was characterized (Dong et al., 2021). The BK D434G mutation mice manifested symptoms resembling the human patients affected by the BK D434G mutation. Simultaneous video-EEG recordings showed that the BK-D434G mice had frequent episodes of spontaneous, generalized SWDs, and during which behavioral arrests, characteristic of absence seizures. These mice were also more susceptible to the induction of generalized seizures by injection of low dose of pentylenetetrazole (PTZ), a convulsant, than the WT mice, consistent with that some BKD434G patients developed generalized tonic-clonic seizures ( Du et al., 2005). Consistent with the human patients, the mutant animals exhibited severe locomotive defects in a battery of motor tests when there was no absence seizure. However, due to the limitations of mouse models, it is difficult to clearly tell if the animals also had PNKD. Both the heterozygous BK-D434G mutation $\left(\mathrm{BK}^{D G / W T}\right)$ and the homozygous mutation $\left(\mathrm{BK}^{D G / D G}\right)$ mice exhibited these symptoms, but the incidents were more frequent or severe in $\mathrm{BK}^{D G / D G}$ mice. All these symptoms could be suppressed by the treatment with a $\mathrm{BK}$ channel inhibitor, paxilline (PAX). These results support the idea that the D434G mutation in BK channels causes a gain of function of the channel that results in epilepsy and movement disorders.

Acute brain slice recordings from the BK D434G mutation mice further showed that the mutation caused hyperexcitability in both the cortical pyramidal neurons and the cerebellar Purkinje cells (Dong et al., 2021), which play essential roles in the pathogenesis of absence seizures (Crunelli et al., 2020) and contribute to the motor defects (Sausbier et al., 2004), respectively. These neurons from the $\mathrm{BK}^{D G / W T}$ mice exhibited a significantly increased action potential frequency compared with the $\mathrm{BK}^{W T / W T}$ mice. The action potentials in $\mathrm{BK}^{D G / W T}$ neurons showed a much faster repolarization with a shortened duration and augmented after-hyperpolarization amplitude (AHP). These results are consistent with that the BK-D434G channels have a higher $\mathrm{Ca}^{2+}$ sensitivity (Díez-Sampedro et al., 2006; Wang et al., 2009; Yang et al., 2010), allowing the channels to activate more during an action potential following membrane depolarization and the opening of voltage gated $\mathrm{Ca}^{2+}$ channels. The enhanced $\mathrm{BK}$ currents more efficiently hyperpolarize the membrane, enabling a faster recovery of the voltage-gated sodium channels from inactivation and potentially facilitating the activation of the hyperpolarization-activated cation $(\mathrm{HCN})$ channels (Lancaster and Nicoll, 1987; Storm, 1987; Shah, 2014), which allow the neurons fire at a higher frequency. The application of BK channel 

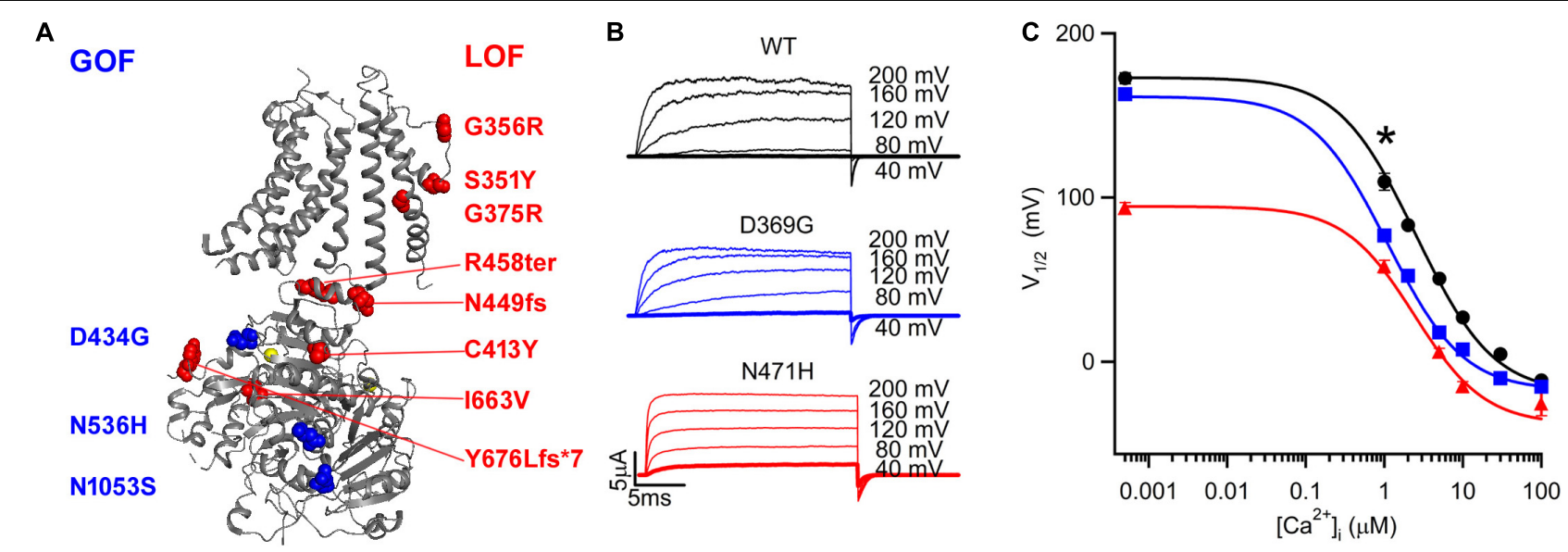

FIGURE 3 | BK mutations alter activation and link to neurological diseases. (A) The GOF (blue) and LOF (red) mutations mapped onto Slo1 structure. Yellow circles: $\mathrm{Ca}^{2+}$ ions bound to the channel. (B) The GOF mutation D434G and N536H in human Slo1 corresponds to D369G and N471H in mouse Slo1, respectively. Both mutations enhance currents at the same voltage and $\mathrm{Ca}^{2+}$ concentration. The current trace at $+40 \mathrm{mV}$, which is most relevant physiological membrane potential, is highlighted in a thicker line. (C) $\mathrm{Ca}^{2+}$ dependence of the $\mathrm{V}_{1 / 2}$ of $\mathrm{GV}$ relations (the voltage where $\mathrm{GV}$ relation is half maximum, which is used to measure the voltage range in which the channel activates). The result shows that with the D369G and N471H mutations GV shifts to more negative voltages at all Ca ${ }^{2+}$ concentrations. The * shows that $1 \mu \mathrm{M} \mathrm{Ca}^{2+}$ concentration is most physiologically relevant. The same results have been published previously (Yang et al., 2010; Zhang et al., 2020).

inhibitor PAX widened the action potential, reduced AHP and decreased firing frequency.

The seizures in patients affected by BK D434G were responsive to general anti-epilepsy medicine (Du et al., 2005), and so were in the BK D434G mutation mice (Dong et al., 2021). Both ethosuximide and valproate suppressed generalized SWDs (Dong et al., 2021). It was shown that the anti-epilepsy medicine acetazolamide had no direct effect on BK channels (Moldenhauer et al., 2020), suggesting that BK channels were not the target of these general anti-epilepsy medicines. It is reported that $30 \%$ of absence epilepsy patients are pharmaco-resistant with the firstline anti-absence medicines (Crunelli et al., 2020). The results of BK channel inhibitor PAX (Dong et al., 2021) suggest that BK channels as a new target for anti-absence therapy may be developed to treat those pharmaco-resistant patients. However, the effects of PAX in suppressing generalized SWDs in BK D434G mutation mice only lasted for $\sim 30 \mathrm{~min}$, which indicates that novel BK channel inhibitors with appropriate efficacy, specificity and pharmacokinetics need to be developed. The development of such novel drugs will benefit from the understanding of molecular mechanisms of BK channel gating.

\section{Neurological Diseases Associated With Other BK Channel Gain of Function Mutations}

The second disease associated KCNMA1 variant was identified in 2015, which resulted in the N1053S mutation in Slo1 (Zhang et al., 2015; Figure 3A). Subsequently more patients with the same Slo1 mutation were identified [also called N995S (Li et al., 2018), or N999S (Heim et al., 2020)]. These mutations were all de novo in unrelated patients. Some of these patients suffered movement disorders, diagnosed with PNKD (Zhang et al., 2015) or cataplexy (Heim et al., 2020), while some only suffered absence epilepsy or mixed with myoclonic seizures (Li et al., 2018). Only one patient suffered both symptoms (Heim et al., 2020). These patients also showed various degrees of developmental delay or intellectual disability. Whether symptoms respond to anti-epileptic medicines also varied among these patients.

As the symptoms of BK-N1053S patients showed some resemblance to those of BK-D434G patients, N1053S mutation is also a gain of function (GOF) mutation. Similar to D434G, the N1053S mutation increased BK currents and the activation kinetics at physiological intracellular $\mathrm{Ca}^{2+}$ concentrations with all depolarizing voltages ( $\mathrm{Li}$ et al., 2018; Moldenhauer et al., 2020), suggesting that the mutation in neurons would shorten action potentials, increase after hyperpolarization and enhance spike firing frequencies (Li et al., 2018). However, the molecular mechanism of N1053S in causing functional changes differs from that of D434G. While G434G primarily enhances $\mathrm{Ca}^{2+}$ sensitivity, N1053S does not seem to alter $\mathrm{Ca}^{2+}$ dependent activation. The G-V relation of the N1053S BK channels shifts to negative voltages with a similar amount at different $\mathrm{Ca}^{2+}$ concentrations, and the deletion of $\mathrm{Ca}^{2+}$ binding sites does not prevent the G-V shift of the N1053S BK channels (Li et al., 2018).

Recently another de novo GOF KCNMA1 variant that resulted in a Slo1 N536H mutation (Figure 3A) was identified, with which the patient suffered frequent dystonic/atonic spells (Zhang et al., 2020). She also had disorders of autism spectrum, attention deficit hyperactivity, and intellectual disability. No obvious seizure was observed in the patients. The symptoms were not responsive to antiepileptic medicines, but dextroamphetamine, a central nervous system stimulant, completely controlled her dystonic episodes from $>100$ per day to none. $\mathrm{N} 536 \mathrm{H}$ is also a GOF mutation that increases $\mathrm{BK}$ currents at $1 \mu \mathrm{M} \mathrm{Ca}^{2+}$ concentration by shifting $\mathrm{G}-\mathrm{V}$ relation to negative voltages (Figures 3B,C). Similar to N1053S, but unlike G434G, N536H does not alter $\mathrm{Ca}^{2+}$ dependent activation of the BK channel. 
The findings so far suggest that the BK channel GOF mutations are associated with absence epilepsy with possible development of myoclonic seizures, movement disorders, or both symptoms. However, it needs to be emphasized that these symptoms vary from patient to patient. The patients may also have cerebellar atrophy, development delays, autism spectrum and intellectual disability to various degrees. These different symptoms derive from increased BK currents either due to increased $\mathrm{Ca}^{2+}$ sensitivity or shift of $\mathrm{G}-\mathrm{V}$ relation with an intact $\mathrm{Ca}^{2+}$ dependent activation. The larger BK currents may result in hyperactivity in different neuron types to cause various symptoms. Future studies are needed to dissect how the GOF BK channel mutations with increased $\mathrm{Ca}^{2+}$ sensitivity or shift of $\mathrm{G}-\mathrm{V}$ relation with an intact $\mathrm{Ca}^{2+}$ dependent activation lead to different neurological symptoms.

\section{Neurological Diseases Associated With BK Channel Loss of Function Mutations}

A report in Tabarki et al. (2016) described two sisters harboring a homozygous KCNMA1 variant that resulted in the frameshift mutation T676Lfs*7, which changed T676 to $\mathrm{L}$ and caused a frame shift after 7 residues downstream (Figure 3A). The patients suffered development delays, severe cerebellar atrophy and seizures of myoclonic type, which progressed to tonic seizures with one of the siblings. The homozygous frameshift mutation presumably abolished the BK channel function although no functional studies were conducted. Several other loss of function (LOF) de novo $\mathrm{BK}$ channel mutations that either completely destroyed BK channel function or reduce BK channel currents were subsequently identified in unrelated patients. The mutations that destroyed BK channel function included G375R, S351Y, G356R, N449fs, I663V (Liang et al., 2019) and the truncation mutation R458ter (Yesil et al., 2018). Other mutations, including C413Y and P805L, decrease BK currents either by reduced protein expression or a shift of the $\mathrm{G}-\mathrm{V}$ relation to the positive voltages (Liang et al., 2019; Figure 3A). These patients suffered neurological diseases that vary in symptoms and severity, most had development delay, intellectual disability, movements disorders (such as ataxia and axial hypotonia), and cerebellar atrophy. Some of the patients also had seizures.

These results reveal that the normal neurological functions are sensitive to $\mathrm{BK}$ channel function. Either a GOF or LOF BK channel mutation could tip the balance of neural function and lead to neurological diseases. While the symptoms of both GOF and LOF BK channel mutations show some overlap, such as development delay, intellectual disability, movement disorder, and epilepsy, the LOF BK channel mutations result in more severe cerebellar atrophy. The patients with BK G375R mutation even had visceral and cardiac malformations, connective tissue symptoms, and dysmorphic features, suggesting that BK channel mutations can impact organs beyond the nervous system (Liang et al., 2019). In a recent study a BK LOF mutation, G354S (Figure 3A), was identified in association with cerebellar degeneration, ataxia, developmental delay and intellectual disability in a young girl (Du et al., 2020). The mutation caused a shift of $\mathrm{G}-\mathrm{V}$ relation of $\mathrm{BK}$ channel activation to more negative voltages. However, due to its location in the selectivity filter of the channel the G354S mutation reduced single channel conductance and increased $\mathrm{Na}^{+}$permeability, thereby decreasing the macroscopic currents and making the currents less effective in repolarizing the membrane potential. Viral transfection of the G354S BK in mouse brain induced ataxia in the animals. Transfection of the G354S BK into dividing PC12 cells, the mutant channel suppressed outgrowth of neurites by reducing the neurite length. The mutant BK channel was also toxic to mitochondria, with the cells expressing the G354S BK showing reduced mitochondria content, disrupted mitochondria superstructure, altered mitochondria dynamics to increase fragmented fission forms, and decreased mitochondria membrane potential. NS1619, a BK channel activator, protected the cells expressing G354S BK from the reduced neurite outgrowth, cell death, and changes in mitochondria, suggesting that the recovery of BK channel function can be a therapy for the neurological diseases associated with BK channel LOF mutations.

\section{CONCLUDING REMARKS}

In ion channel activation sensors change conformation upon physical or chemical stimulation, which induces pore opening via interactions between sensors and the pore known as sensor-pore coupling. BK channel activation has shown unique characteristics in all these molecular steps as compared to canonical activation mechanisms in voltage gated $\mathrm{K}^{+}(\mathrm{Kv})$ channels. First, BK channels sense both membrane voltage and intracellular $\mathrm{Ca}^{2+}$ with distinct voltage sensor and $\mathrm{Ca}^{2+}$ binding sites. For these two stimuli to control the opening of the same pore independently the sensors are coupled with the pore with allosteric mechanisms. Second, the VSD and PGD domains in BK channels are not domain swapped among subunits, which differs from most $\mathrm{Kv}$ channels, and thus the VSD-pore coupling mechanism in BK channels is also unique. Third, the activation gate in BK channels is not a physical barrier at the intracellular side of the pore, but may be a vaper barrier as in a hydrophobic gating mechanism.

Mutations of BK channels that either increase BK channel currents (GOF) or decrease currents (LOF) at physiological voltages and $\mathrm{Ca}^{2+}$ concentrations are associated with neurological disorders. The changes in BK channel currents alter excitability of various neurons that may induce the symptoms. The LOF BK mutations may also directly disrupt mitochondria functions and cause cell death in brain and other organs. In the future, it is critical to translate the comprehensive understanding of BK channel structure and function to uncover the neurological mechanisms of BK channelopathy and design precision therapies to treat these patients. A key question is how the mutations of BK channels that either increase BK channel currents (GOF) or decrease currents (LOF) at physiological voltages and $\mathrm{Ca}^{2+}$ concentrations are associated with such a wide spectrum of neurological disorders. Animal models carrying different $\mathrm{BK}$ channel mutations and induced pluripotent stem cells (iPSCs) directly derived from patients are invaluable tools to address this question.

On the other hand, the change of voltage and $\mathrm{Ca}^{2+}$ dependent activation by the disease-associating mutations also provide 
unique insights for further understanding of BK molecular mechanisms. For instance, while we can reason that G375R (Liang et al., 2019; Figure 3A) may disrupt the opening of the activation gate since this is part of the diglycine hinge for the BK channel activation gate (Magidovich and Yifrach, 2004) and G356R may destroy the selectivity filter (Tao and MacKinnon, 2019; Figure 3A), we have no clue how the mutations in the cytosolic domain, such as N1053S (Zhang et al., 2015), $\mathrm{N} 536 \mathrm{H}$ (Zhang et al., 2020), C413Y, or I663V (Liang et al., 2019; Figure 3A) may affect voltage dependent activation or the intrinsic gate opening in BK channels. Further studies of these mutations may provide insights to how the CTD interacts with VSD and PGD to control BK channel activation.

The studies of human genetics on BK channelopathy, biophysical characterizations, and the animal models carrying either GOF or LOF BK channel mutations indicate that BK channels can be a promising drug target for treating associated neurological diseases (Du et al., 2020; Dong et al., 2021). The

\section{REFERENCES}

Adelman, J. P., Shen, K. Z., Kavanaugh, M. P., Warren, R. A., Wu, Y. N., Lagrutta, A., et al. (1992). Calcium-activated potassium channels expressed from cloned complementary DNAs. Neuron 9, 209-216. doi: 10.1016/0896-6273(92) 90160-F

Aggarwal, S. K., and MacKinnon, R. (1996). Contribution of the S4 segment to gating charge in the Shaker K+ channel. Neuron 16, 1169-1177. doi: 10.1016/ S0896-6273(00)80143-9

Aryal, P., Sansom, M. S. P., and Tucker, S. J. (2015). Hydrophobic gating in ion channels. J. Mol. Biol. 427, 121-130. doi: 10.1016/j.jmb.2014.07.030

Atkinson, N. S., Robertson, G. A., and Ganetzky, B. (1991). A component of calcium-activated potassium channels encoded by the Drosophila slo locus. Science 253, 551-555. doi: 10.1126/science.1857984

Bailey, C. S., Moldenhauer, H. J., Park, S. M., Keros, S., and Meredith, A. L. (2019). KCNMA1-linked channelopathy. J. Gen. Physiol. 151, 1173-1189. doi: 10.1085/jgp.201912457

Bao, L., Kaldany, C., Holmstrand, E. C., and Cox, D. H. (2004). Mapping the BKCa channel's "Ca2+ bowl": side-chains essential for Ca2+ sensing. J. Gen. Physiol. 123, 475-489. doi: 10.1085/jgp.200409052

Bezanilla, F. (2000). The voltage sensor in voltage-dependent ion channels. Physiol. Rev. 80, 555-592. doi: 10.1152/physrev.2000.80.2.555

Budelli, G., Geng, Y. Y., Butler, A., Magleby, K. L., and Salkoff, L. (2013). Properties of Slo1 K+ channels with and without the gating ring. Proc. Natl. Acad. Sci. U.S.A. 110, 16657-16662. doi: 10.1073/pnas.1313433110

Butler, A., Tsunoda, S., McCobb, D. P., Wei, A., and Salkoff, L. (1993). mSlo, a complex mouse gene encoding "maxi” calcium-activated potassium channels. Science 261, 221-224. doi: 10.1126/science.7687074

Chen, X., Yan, J., and Aldrich, R. W. (2014). BK channel opening involves sidechain reorientation of multiple deep-pore residues. Proc. Natl. Acad. Sci. U.S.A. 111, E79-E88. doi: 10.1073/pnas.1321697111

Cowgill, J., and Chanda, B. (2021). Mapping electromechanical coupling pathways in voltage-gated ion channels: challenges and the way forward. J. Mol. Biol. 433:167104. doi: 10.1016/j.jmb.2021.167104

Cox, D. H., Cui, J., and Aldrich, R. W. (1997). Allosteric gating of a large conductance Ca-activated K+ channel. J. Gen. Physiol. 110, 257-281. doi: 10.1085/jgp.110.3.257

Crunelli, V., Lőrincz, M. L., McCafferty, C., Lambert, R. C., Leresche, N., Di Giovanni, G., et al. (2020). Clinical and experimental insight into pathophysiology, comorbidity and therapy of absence seizures. Brain 143, 2341-2368. doi: 10.1093/brain/awaa072

Cui, J. (2010). BK-type calcium-activated potassium channels: coupling of metal ions and voltage sensing. J. Physiol. 588, 4651-4658. doi: 10.1113/jphysiol.2010. 194514 studies of molecular mechanisms of BK channel activation and neurological diseases associated with aberrant BK channel function are valuable in directing the diagnoses of such diseases and the development of BK channel specific modulators for the therapy.

\section{AUTHOR CONTRIBUTIONS}

The author confirms being the sole contributor of this work and has approved it for publication.

\section{ACKNOWLEDGMENTS}

The author would like to thank Jianhan Chen and Huanghe Yang for the critical reading of the manuscript. Guohui Zhang helped make the figures and format references.

Cui, J. (2020). The action of a BK channel opener. J. Gen. Physiol. 152:e202012571. doi: 10.1085/jgp.202012571

Cui, J., and Aldrich, R. W. (2000). Allosteric linkage between voltage and Ca2+dependent activation of BK-type mslo1 K+ channels. Biochemistry 39, 1561215619. doi: 10.1021/bi001509+

Cui, J., Cox, D. H., and Aldrich, R. W. (1997). Intrinsic voltage dependence and $\mathrm{Ca} 2+$ regulation of mslo large conductance Ca-activated K+ channels. J. Gen. Physiol. 109, 647-673. doi: 10.1085/jgp.109.5.647

Diaz, L., Meera, P., Amigo, J., Stefani, E., Alvarez, O., Toro, L., et al. (1998). Role of the S4 segment in a voltage-dependent calcium-sensitive potassium (hSlo) channel. J. Biol. Chem. 273, 32430-32436. doi: 10.1074/jbc.273.49.32430

Díez-Sampedro, A., Silverman, W. R., Bautista, J. F., and Richerson, G. B. (2006). Mechanism of increased open probability by a mutation of the BK channel. J. Neurophysiol. 96, 1507-1516. doi: 10.1152/jn.00461.2006

Dong, P., Zhang, Y., Mikati, M. A., Cui, J., and Yang, H. (2021). Neuronal mechanism of a BK channelopathy in absence epilepsy and movement disorders. bioRxiv [Preprint] bioRxiv: 2021.06.30.450615, doi: 10.1101/2021. 06.30 .450615

Du, W., Bautista, J. F., Yang, H., Diez-Sampedro, A., You, S.-A., Wang, L., et al. (2005). Calcium-sensitive potassium channelopathy in human epilepsy and paroxysmal movement disorder. Nat. Genet. 37, 733-738. doi: 10.1038/ ng1585

Du, X., Carvalho-de-Souza, J. L., Wei, C., Carrasquel-Ursulaez, W., Lorenzo, Y., Gonzalez, N., et al. (2020). Loss-of-function BK channel mutation causes impaired mitochondria and progressive cerebellar ataxia. Proc. Natl. Acad. Sci. U.S.A. 117:6023. doi: 10.1073/pnas.1920008117

Gandhi, C. S., and Isacoff, E. Y. (2002). Molecular models of voltage sensing. J. Gen. Physiol. 120, 455-463. doi: 10.1085/jgp.20028678

Geng, Y., Deng, Z., Zhang, G., Budelli, G., Butler, A., Yuan, P., et al. (2020). Coupling of $\mathrm{Ca}(2+)$ and voltage activation in $\mathrm{BK}$ channels through the alphaB helix/voltage sensor interface. Proc. Natl. Acad. Sci. U.S.A. 117, 14512-14521. doi: 10.1073/pnas.1908183117

Heim, J., Vemuri, A., Lewis, S., Guida, B., Troester, M., Keros, S., et al. (2020). Cataplexy in patients harboring the KCNMA1 p.N999S mutation. Mov. Disord. Clin. Pract. 7, 861-862. doi: 10.1002/mdc3.13024

Hite, R. K., Tao, X., and MacKinnon, R. (2017). Structural basis for gating the highconductance Ca2+-activated $\mathrm{K}+$ channel. Nature 541, 52-57. doi: 10.1038/ nature 20775

Horrigan, F. T., and Aldrich, R. W. (1999). Allosteric voltage gating of potassium channels II. Mslo channel gating charge movement in the absence of $\mathrm{Ca} 2+$. J. Gen. Physiol. 114, 305-336. doi: 10.1085/jgp.114.2.305

Horrigan, F. T., and Aldrich, R. W. (2002). Coupling between voltage sensor activation, $\mathrm{Ca} 2+$ binding and channel opening in large conductance (BK) potassium channels. J. Gen. Physiol. 120, 267-305. doi: 10.1085/jgp.20028605 
Horrigan, F. T., Cui, J., and Aldrich, R. W. (1999). ) Allosteric voltage gating of potassium channels I. Mslo ionic currents in the absence of Ca2+. J. Gen. Physiol. 114, 277-304. doi: 10.1085/jgp.114.2.277

Hou, P., Kang, P. W., Kongmeneck, A. D., Yang, N. D., Liu, Y., Shi, J., et al. (2020). Two-stage electro-mechanical coupling of a KV channel in voltage-dependent activation. Nat. Commun. 11:676. doi: 10.1038/s41467-020-14406-w

Hu, L., Shi, J., Ma, Z., Krishnamoorthy, G., Sieling, F., Zhang, G., et al. (2003). Participation of the S4 voltage sensor in the Mg2+-dependent activation of large conductance (BK) K+ channels. Proc. Natl. Acad. Sci. U.S.A. 100, 10488-10493. doi: $10.1073 /$ pnas. 1834300100

Islas, L. D., and Sigworth, F. J. (1999). Voltage sensitivity and gating charge in shaker and shab family potassium channels. J. Gen. Physiol. 114, 723-742. doi: 10.1085/jgp.114.5.723

Javaherian, A. D., Yusifov, T., Pantazis, A., Franklin, S., Gandhi, C. S., and Olcese, R. (2011). Metal-driven operation of the human large-conductance voltage- and Ca2+-dependent potassium channel (BK) gating ring apparatus. J. Biol. Chem. 286, 20701-20709. doi: 10.1074/jbc.M111.235234

Jia, Z., Yazdani, M., Zhang, G., Cui, J., and Chen, J. (2018). Hydrophobic gating in BK channels. Nat. Commun. 9:3408. doi: 10.1038/s41467-018-05970-3

Jiang, Y., Lee, A., Chen, J., Cadene, M., Chait, B. T., and MacKinnon, R. (2002a). Crystal structure and mechanism of a calcium-gated potassium channel. Nature 417, 515-522. doi: 10.1038/417515a

Jiang, Y., Lee, A., Chen, J., Cadene, M., Chait, B. T., and MacKinnon, R. (2002b), The open pore conformation of potassium channels. Nature 417, 523-526. doi: $10.1038 / 417523 a$

Kaczorowski, G. J., and Garcia, M. L. (2016). "Chapter twelve - developing molecular pharmacology of bk channels for therapeutic benefit," in International Review of Neurobiology, ed. C. Contet (Cambridge, MA: Academic Press), 439-475. doi: 10.1016/bs.irn.2016.02.013

Krishnamoorthy, G., Shi, J., Sept, D., and Cui, J. (2005). The NH2 terminus of RCK1 domain regulates $\mathrm{Ca} 2+-$ dependent $\mathrm{BK}(\mathrm{Ca})$ channel gating. J. Gen. Physiol. 126, 227-241. doi: 10.1085/jgp.200509321

Lancaster, B., and Nicoll, R. A. (1987). Properties of two calcium-activated hyperpolarizations in rat hippocampal neurones. J. Physiol. (Lond.) 389, 187203. doi: 10.1113/jphysiol.1987.sp016653

Latorre, R., Oberhauser, A., Labarca, P., and Alvarez, O. (1989). Varieties of calcium-activated potassium channels. Annu. Rev. Physiol. 51, 385-399. doi: 10.1146/annurev.ph.51.030189.002125

Lee, U. S., and Cui, J. (2009). \{beta\} subunit-specific modulations of BK channel function by a mutation associated with epilepsy and dyskinesia. J. Physiol. 587, 1481-1498. doi: 10.1113/jphysiol.2009.169243

Lee, U. S., and Cui, J. (2010). BK channel activation: structural and functional insights. Trends Neurosci. 33, 415-423. doi: 10.1016/j.tins.2010. 06.004

Li, W., and Aldrich, R. W. (2004). Unique inner pore properties of BK channels revealed by quaternary ammonium block. J. Gen. Physiol. 124, 43-57. doi: 10.1085/jgp. 200409067

Li, W., and Aldrich, R. W. (2006). State-dependent block of BK channels by synthesized shaker ball peptides. J. Gen. Physiol. 128, 423-441. doi: 10.1085/ jgp.200609521

Li, X., Poschmann, S., Chen, Q., Fazeli, W., Oundjian, N. J., SnoeijenSchouwenaars, F. M., et al. (2018). De novo BK channel variant causes epilepsy by affecting voltage gating but not Ca2+ sensitivity. Eur. J. Hum. Genet. 26, 220-229. doi: 10.1038/s41431-017-0073-3

Liang, L., Li, X., Moutton, S., Schrier Vergano, S. A., Cogné, B., Saint-Martin, A., et al. (2019). De novo loss-of-function KCNMA1 variants are associated with a new multiple malformation syndrome and a broad spectrum of developmental and neurological phenotypes. Hum. Mol. Genet. 28, 2937-2951. doi: 10.1093/ $\mathrm{hmg} / \mathrm{ddz} 117$

Liu, Y., Holmgren, M., Jurman, M. E., and Yellen, G. (1997). Gated access to the pore of a voltage-dependent K+ channel. Neuron 19, 175-184. doi: 10.1016/ S0896-6273(00)80357-8

Long, S. B., Campbell, E. B., and Mackinnon, R. (2005a). Crystal structure of a mammalian voltage-dependent Shaker family K+ channel. Science 309, 897903. doi: $10.1126 /$ science. 1116269

Long, S. B., Campbell, E. B., and Mackinnon, R. (2005b). Voltage sensor of Kv1.2: structural basis of electromechanical coupling. Science 309, 903-908. doi: 10. $1126 /$ science. 1116270
Lorenzo-Ceballos, Y., Carrasquel-Ursulaez, W., Castillo, K., Alvarez, O., and Latorre, R. (2019). Calcium-driven regulation of voltage-sensing domains in BK channels. eLife 8:e44934. doi: 10.7554/eLife.44934.018

$\mathrm{Lu}, \mathrm{Z}$., Klem, A. M., and Ramu, Y. (2001). Ion conduction pore is conserved among potassium channels. Nature 413, 809-813. doi: $10.1038 / 35101535$

Ma, Z. M., and Horrigan, F. T. (2005). Voltage-sensing residues in S2 and S4 segments of the BK channel. Biophys. J. 88:100A.

Magidovich, E., and Yifrach, O. (2004). Conserved gating hinge in ligand- and voltage-dependent $\mathrm{K}+$ channels. Biochemistry 43, 13242-13247. doi: 10.1021/ bi $048377 \mathrm{v}$

Mannuzzu, L. M., Moronne, M. M., and Isacoff, E. Y. (1996). Direct physical measure of conformational rearrangement underlying potassium channel gating. Science 271, 213-216. doi: 10.1126/science.271.5246.213

Marty, A. (1981). Ca-dependent K channels with large unitary conductance in chromaffin cell membranes. Nature 291, 497-500. doi: 10.1038/291497a0

McManus, O. B., and Magleby, K. L. (1991). Accounting for the Ca2+-dependent kinetics of single large-conductance $\mathrm{Ca} 2+$-activated $\mathrm{K}+$ channels in rat skeletal muscle. J. Physiol. 443, 739-777. doi: 10.1113/jphysiol.1991.sp018861

Miller, J. P., Moldenhauer, H. J., Keros, S., and Meredith, A. L. (2021). Emerging spectrum of variants and clinical features in KCNMA1-linked channelopathy. Channels 15, 447-464. doi: 10.1080/19336950.2021.1938852

Miranda, P., Holmgren, M., and Giraldez, T. (2018). Voltage-dependent dynamics of the BK channel cytosolic gating ring are coupled to the membrane-embedded voltage sensor. eLife 7:e40664. doi: 10.7554/eLife.40664.014

Moldenhauer, H. J., Matychak, K. K., and Meredith, A. L. (2020). Comparative gain-of-function effects of the KCNMA1-N999S mutation on human BK channel properties. J. Neurophysiol. 123, 560-570. doi: 10.1152/jn.00626.2019

Niu, X., Qian, X., and Magleby, K. L. (2004). Linker-gating ring complex as passive spring and $\mathrm{Ca} 2+-$ dependent machine for a voltage- and Ca2+-activated potassium channel. Neuron 42, 745-756. doi: 10.1016/j.neuron.2004.05.001

Pallotta, B. S., Magleby, K. L., and Barrett, J. N. (1981). Single channel recordings of Ca2+-activated K+ currents in rat muscle cell culture. Nature 293, 471-474. doi: 10.1038/293471a0

Pantazis, A., and Olcese, R. (2012). Relative transmembrane segment rearrangements during BK channel activation resolved by structurally assigned fluorophore-quencher pairing. J. Gen. Physiol. 140, 207-218. doi: 10.1085/jgp. 201210807

Pantazis, A., Gudzenko, V., Savalli, N., Sigg, D., and Olcese, R. (2010a). Operation of the voltage sensor of a human voltage- and $\mathrm{Ca}^{2+}$-activated $\mathrm{K}^{+}$channel. Proc. Natl. Acad. Sci. U.S.A. 107, 4459-4464. doi: 10.1073/pnas.0911959107

Pantazis, A., Kohanteb, A. P., and Olcese, R. (2010b). Relative motion of transmembrane segments S0 and S4 during voltage sensor activation in the human BK(Ca) channel. J. Gen. Physiol. 136, 645-657. doi: 10.1085/jgp. 201010503

Pantazis, A., Westerberg, K., Althoff, T., Abramson, J., and Olcese, R. (2018). Harnessing photoinduced electron transfer to optically determine protein subnanoscale atomic distances. Nat. Commun. 9: 4738. doi: 10.1038/s41467-01807218-6

Rockman, M. E., Vouga, A. G., and Rothberg, B. S. (2020). Molecular mechanism of BK channel activation by the smooth muscle relaxant NS11021. J. Gen. Physiol. 152:e201912506. doi: 10.1085/jgp.201912506

Sausbier, M., Hu, H., Arntz, C., Feil, S., Kamm, S., Adelsberger, H., et al. (2004). Cerebellar ataxia and Purkinje cell dysfunction caused by $\mathrm{Ca} 2+$-activated $\mathrm{K}+$ channel deficiency. Proc. Natl. Acad. Sci. U.S.A. 101, 9474-9478. doi: 10.1073/ pnas. 0401702101

Savalli, N., Kondratiev, A., Toro, L., and Olcese, R. (2006). Voltage-dependent conformational changes in human $\mathrm{Ca}(2+)$ - and voltage-activated $\mathrm{K}(+)$ channel, revealed by voltage-clamp fluorometry. Proc. Natl. Acad. Sci. U.S.A. 103, 1261912624. doi: $10.1073 /$ pnas. 0601176103

Savalli, N., Pantazis, A., Yusifov, T., Sigg, D., and Olcese, R. (2012). The contribution of RCK domains to human BK channel allosteric activation. J. Biol. Chem. 287, 21741-21750. doi: 10.1074/jbc.M112.346171

Schewe, M., Sun, H., Mert, U., Mackenzie, A., Pike, A. C. W., Schulz, F., et al. (2019). A pharmacological master key mechanism that unlocks the selectivity filter gate in $\mathrm{K}(+)$ channels. Science 363, 875-880. doi: 10.1126/science.aav 0569

Schreiber, M., and Salkoff, L. (1997). A novel calcium-sensing domain in the BK channel. Biophys. J. 73, 1355-1363. doi: 10.1016/S0006-3495(97)78168-2 
Seoh, S. A., Sigg, D., Papazian, D. M., and Bezanilla, F. (1996). Voltage-sensing residues in the S2 and S4 segments of the Shaker K+ channel. Neuron 16, 1159-1167. doi: 10.1016/S0896-6273(00)80142-7

Shah, M. M. (2014). Cortical HCN channels: function, trafficking and plasticity. J. Physiol. 592, 2711-2719. doi: 10.1113/jphysiol.2013.270058

Shi, J., and Cui, J. (2001). Intracellular Mg2+ enhances the function of BK-type Ca2+-activated K+ channels. J. Gen. Physiol. 118, 589-606. doi: 10.1085/jgp. 118.5.589

Shi, J., Krishnamoorthy, G., Yang, Y., Hu, L., Chaturvedi, N., Harilal, D., et al. (2002). Mechanism of magnesium activation of calcium-activated potassium channels. Nature 418, 876-880. doi: 10.1038/nature00941

Storm, J. F. (1987). Action potential repolarization and a fast afterhyperpolarization in rat hippocampal pyramidal cells. J. Physiol. (Lond.) 385, 733-759. doi: 10.1113/jphysiol.1987.sp016517

Sweet, T.-B., and Cox, D. H. (2008). Measurements of the BKCa channel's highaffinity Ca2+ binding constants: effects of membrane voltage. J. Gen. Physiol. 132, 491-505. doi: 10.1085/jgp.200810094

Tabarki, B., AlMajhad, N., AlHashem, A., Shaheen, R., and Alkuraya, F. S. (2016). Homozygous KCNMA1 mutation as a cause of cerebellar atrophy, developmental delay and seizures. Hum. Genet. 135, 1295-1298. doi: 10.1007/ s00439-016-1726-y

Tang, Q.-Y., Zeng, X.-H., and Lingle, C. J. (2009). Closed-channel block of BK potassium channels by bbTBA requires partial activation. J. Gen. Physiol. 134, 409-436. doi: 10.1085/jgp.200910251

Tao, X., and MacKinnon, R. (2019). Molecular structures of the human Slo1 K(+) channel in complex with beta4. eLife 8:e51409. doi: 10.7554/eLife.51409

Tao, X., Hite, R. K., and MacKinnon, R. (2017). Cryo-EM structure of the open high-conductance Ca2+-activated $\mathrm{K}+$ channel. Nature 541, 46-51. doi: 10. 1038/nature20608

Tian, Y., Heinemann, S. H., and Hoshi, T. (2019). Large-conductance $\mathrm{Ca}^{2+}$ - and voltage-gated $\mathrm{K}^{+}$channels form and break interactions with membrane lipids during each gating cycle. Proc. Natl. Acad. Sci. U.S.A. 116, 8591-8596. doi: 10.1073/pnas.1901381116

Wang, B., Rothberg, B. S., and Brenner, R. (2009). Mechanism of increased BK channel activation from a channel mutation that causes epilepsy. J. Gen. Physiol. 133, 283-294. doi: 10.1085/jgp.200810141

Wilkens, C. M., and Aldrich, R. W. (2006). State-independent block of BK channels by an intracellular quaternary ammonium. J. Gen. Physiol. 128, 347-364.

Wu, Y., Yang, Y., Ye, S., and Jiang, Y. (2010). Structure of the gating ring from the human large-conductance $\mathrm{Ca}(2+)$-gated $\mathrm{K}(+)$ channel. Nature 466, 393-397.

Xia, X.-M., Zeng, X., and Lingle, C. J. (2002). Multiple regulatory sites in largeconductance calcium-activated potassium channels. Nature 418, 880-884.

Yang, H., Hu, L., Shi, J., Delaloye, K., Horrigan, F. T., and Cui, J. (2007). Mg2+ mediates interaction between the voltage sensor and cytosolic domain to activate BK channels. Proc. Natl. Acad. Sci. U.S.A. 104, 18270-18275.

Yang, H., Shi, J., Zhang, G., Yang, J., Delaloye, K., and Cui, J. (2008). Activation of Slo1 BK channels by $\mathrm{Mg} 2+$ coordinated between the voltage sensor and RCK1 domains. Nat. Struct. Mol. Biol. 15, 1152-1159.

Yang, J., Krishnamoorthy, G., Saxena, A., Zhang, G., Shi, J., Yang, H., et al. (2010). An epilepsy/dyskinesia-associated mutation enhances BK channel activation by potentiating Ca2+ sensing. Neuron 66, 871-883.

Yang, J., Yang, H., Sun, X., Delaloye, K., Yang, X., Moller, A., et al. (2013). Interaction between residues in the $\mathrm{Mg} 2+$-binding site regulates $\mathrm{BK}$ channel activation. J. Gen. Physiol. 141, 217-228.

Yazdani, M., Zhang, G., Jia, Z., Shi, J., Cui, J., and Chen, J. (2020b). Aromatic interactions with membrane modulate human BK channel activation. eLife 9:e55571.

Yazdani, M., Jia, Z., and Chen, J. (2020a). Hydrophobic dewetting in gating and regulation of transmembrane protein ion channels. J. Chem. Phys. 153: 110901.

Yesil, G., Aralasmak, A., Akyuz, E., Icagasioglu, D., Uygur Sahin, T., and Bayram, Y. (2018). Expanding the phenotype of homozygous KCNMA1 mutations; dyskinesia, epilepsy, intellectual disability, cerebellar and corticospinal tract atrophy. Balkan Med. J. 35, 336-339.
Yuan, P., Leonetti, M. D., Hsiung, Y., and MacKinnon, R. (2012). Open structure of the $\mathrm{Ca} 2+$ gating ring in the high-conductance $\mathrm{Ca} 2+$-activated $\mathrm{K}+$ channel. Nature 481, 94-97. doi: 10.1038/nature10670

Yuan, P., Leonetti, M. D., Pico, A. R., Hsiung, Y., and MacKinnon, R. (2010). Structure of the human BK channel Ca2+-activation apparatus at $3.0 \mathrm{~A}$ resolution. Science 329, 182-186. doi: 10.1126/science.1190414

Yusifov, T., Savalli, N., Gandhi, C. S., Ottolia, M., and Olcese, R. (2008). The RCK2 domain of the human BKCa channel is a calcium sensor. Proc. Natl. Acad. Sci. U.S.A. 105, 376-381. doi: 10.1073/pnas.0705261105

Zagotta, W. N., Hoshi, T., and Aldrich, R. W. (1994a). Shaker potassium channel gating. III: evaluation of kinetic models for activation. J. Gen. Physiol. 103, 321-362. doi: 10.1085/jgp.103.2.321

Zagotta, W. N., Hoshi, T., Dittman, J., and Aldrich, R. W. (1994b). Shaker potassium channel gating. II: transitions in the activation pathway. J. Gen. Physiol. 103, 279-319. doi: 10.1085/jgp.103.2.279

Zeng, X. H., Xia, X. M., and Lingle, C. J. (2005). Divalent cation sensitivity of BK channel activation supports the existence of three distinct binding sites. J. Gen. Physiol. 125, 273-286. doi: 10.1085/jgp.200409239

Zhang, G., Geng, Y., Jin, Y., Shi, J., McFarland, K., Magleby, K. L., et al. (2017). Deletion of cytosolic gating ring decreases gate and voltage sensor coupling in BK channels. J. Gen. Physiol. 149, 373-387. doi: 10.1085/jgp.201611646

Zhang, G., Gibson, R. A., McDonald, M., Liang, P., Kang, P. W., Shi, J., et al. (2020). A gain-of-function mutation in KCNMA1 causes dystonia spells controlled with stimulant therapy. Mov. Disord. 35, 1868-1873. doi: 10.1002/mds. 28138

Zhang, G., Huang, S.-Y., Yang, J., Shi, J., Yang, X., Moller, A., et al. (2010). Ion sensing in the RCK1 domain of BK channels. Proc. Natl. Acad. Sci. U.S.A. 107, 18700-18705. doi: 10.1073/pnas.1010124107

Zhang, G., Yang, H., Liang, H., Yang, J., Shi, J., McFarland, K., et al. (2014). A charged residue in $\mathrm{S} 4$ regulates coupling among the activation gate, voltage, and Ca2+ sensors in BK channels. J. Neurosci. 34, 12280-12288. doi: 10.1523/ JNEUROSCI.1174-14.2014

Zhang, Z. B., Tian, M. Q., Gao, K., Jiang, Y. W., and Wu, Y. (2015). De novo KCNMA1 mutations in children with early-onset paroxysmal dyskinesia and developmental delay. Mov. Disord. 30, 1290-1292. doi: 10.1002/mds.26216

Zhou, Y., Xia, X. M., and Lingle, C. J. (2020). functionally relevant site for paxilline inhibition of BK channels. Proc. Natl. Acad. Sci. U.S.A. 117, 1021-1026. doi: 10.1073/pnas.1912623117

Zhou, Y., Xia, X.-M., and Lingle, C. J. (2011). Cysteine scanning and modification reveal major differences between $\mathrm{BK}$ channels and $\mathrm{Kv}$ channels in the inner pore region. Proc. Natl. Acad. Sci. U.S.A. 108, 12161-12166. doi: 10.1073/pnas. 1104150108

Zhou, Y., Xia, X.-M., and Lingle, C. J. (2015). Cadmium-cysteine coordination in the BK inner pore region and its structural and functional implications. Proc. Natl. Acad. Sci. U.S.A. 112:5237. doi: 10.1073/pnas.1500953112

Conflict of Interest: The author declares that the research was conducted in the absence of any commercial or financial relationships that could be construed as a potential conflict of interest.

The reviewer YZ declared a shared affiliation, with no collaboration, with one of the authors JC to the handling editor at the time of the review.

Publisher's Note: All claims expressed in this article are solely those of the authors and do not necessarily represent those of their affiliated organizations, or those of the publisher, the editors and the reviewers. Any product that may be evaluated in this article, or claim that may be made by its manufacturer, is not guaranteed or endorsed by the publisher.

Copyright (C) 2021 Cui. This is an open-access article distributed under the terms of the Creative Commons Attribution License (CC BY). The use, distribution or reproduction in other forums is permitted, provided the original author(s) and the copyright owner(s) are credited and that the original publication in this journal is cited, in accordance with accepted academic practice. No use, distribution or reproduction is permitted which does not comply with these terms. 